Annales Geophysicae (2001) 19: 699-706 (C) European Geophysical Society 2001

\title{
Estimates of the field-aligned current density in current-carrying filaments using auroral zone ground-based observations
}

\author{
M. A. Danielides ${ }^{1}$, S. Shalimov ${ }^{2}$, and J. Kangas ${ }^{3}$ \\ ${ }^{1}$ Department of Physical Sciences, P.O. Box 3000, FIN-90014 University of Oulu, Finland \\ ${ }^{2}$ Institute of Physics of the Earth, Moscow, Russia \\ ${ }^{3}$ Sodankylä Geophysical Observatory, FIN-99600 Sodankylä, Finland
}

Received: 11 January 2001 - Revised: 25 April 2001 - Accepted: 17 May 2001

\begin{abstract}
We described the ground signatures of dynamic substorm features as observed by the imaging riometer, magnetometers and all-sky camera (ASC) at Kilpisjärvi, Finland on 5 and 25 October 1999 during the late evening hours. The magnetometer data was consistent with the motion of upward field-aligned currents (FACs) associated with absorption patches moving within the field of view of the riometer. We used riometer data in order to estimate the intensity of FACs associated with these local current-carrying filaments. It is shown that during these events, the estimated FAC intensity exceeds a threshold value that corresponds to the excitation of the low-frequency turbulence in the upper ionosphere. As a result, a quasi-oscillating regime of anomalous resistivity on the auroral field lines can give rise to the burstlike electron acceleration responsible for simultaneously observed auroral forms and bursts of Pi1B pulsations.
\end{abstract}

Key words. Ionosphere (active experiments; auroral ionosphere; electric fields and currents

\section{Introduction}

Investigations of field-aligned currents (FACs) in the auroral region are of great interest because it is a well established fact that FACs are responsible for the linkage between magnetospheric and ionospheric plasmas, and FACs are thus important for the understanding of the magnetosphere-ionosphere coupling.

It is also well-known from polar region electrodynamics that the ground magnetic signatures of the ionospheric current systems are solely due to the source-free part of the ionospheric current system (due to Hall currents in the case of a uniform ionospheric conductance distribution (Fukushima, 1976)). This means that the curl-free part of the ionospheric current, together with the FACs, does not produce any magnetic field disturbance below the ionosphere and is thus mag-

Correspondence to: M. A. Danielides

(michael.danielides@oulu.fi) netically invisible on the ground, but can be detected above the ionosphere by satellites.

Since the ground magnetic field disturbances depend on the height-integrated horizontal ionospheric current $\boldsymbol{J}_{\perp}$, and since this current and the electric field in the ionosphere $\boldsymbol{E}_{\perp}$ are connected by Ohm's law via the Hall $\left(\Sigma_{H}\right)$ and Pedersen $\left(\Sigma_{P}\right)$ conductances, it would seem possible then, in principle, by using divergence-free conditions for the total current, to estimate FACs from magnetic and electric field data if, for example, the ratio $\Sigma_{H} / \Sigma_{P}$ is assumed to be known. It is, however, usually difficult to make a quantitative analysis of FACs because there is no simple way to estimate the ionospheric conductivity distribution or the particle flux.

In addition, the precipitation of energetic particles in, for example, auroral arcs can result in small-scale (filamentary) FACs and conductivity gradients. Such filaments which can also be highly variable in time (for example, with a characteristic scale of 30 seconds or less for pulsating aurora) occur within localized regions as small as $1-30 \mathrm{~km}$, i.e. within the auroral arc thickness. Strong conductivity gradients modify the convection-driven electrojets and the associated convection electric fields. However, measurements of electric fields, currents, and conductivities for a localized region of $30 \mathrm{~km}$ or less in dimension and with an appropriate time resolution are rather difficult to obtain by satellites, rockets, and most ground-based experiments.

Ionospheric Hall and Pedersen conductances can be derived, for example, from auroral spectroscopic measurements obtained with meridional scanning photometers (Mende et al., 1984; Robinson et al., 1989). Conductances can also be derived from height profiles of electron density obtained by incoherent scatter radars (see, for example, Schlegel, 1988; Robinson et al., 1989). While a general agreement is obtained by using these basically different methods, some disagreements illustrate the limitations of these experimental techniques. The radar yields accurate values of conductances with limited temporal and spatial resolution, while the optical measurements provide less accurate values with high time resolution and over a large region of space. 
An elegant analytical approach was developed by Inhester et al. (1992) and Amm (1995) (called "method of characteristics") in order to derive ionospheric Hall conductance distribution $\Sigma_{H}$ from ground magnetic and ionospheric electric field observations (hence by estimating $\Sigma_{H} / \Sigma_{P}$ the remaining ionospheric electrodynamical parameters can be inferred).

For the events under study here, however, incoherent scatter radar data was not available for the conductance and electric field measurements, nor were the TV images calibrated in intensity. But two other experiments were available: the Imaging Riometer for Ionospheric Studies (IRIS) (Detrick and Rosenberg, 1990) for ionospheric absorption of cosmic radio noise, and the International Monitor for Auroral Geomagnetic Effects (IMAGE) magnetometer network (Lühr, 1994) for geomagnetic field disturbances and the corresponding equivalent currents. The data from these instruments were used as input parameters in our model calculations, which allowed us to detect and to estimate FAC intensities in current-carrying filaments. Next, we analysed a correlation of the suspected FAC filaments with some additional auroral phenomena observed by ASC and a pulsation magnetometer in order to see in which events the most intense FACs were involved. We estimated from theoretical arguments the threshold value of FAC needed to produce these phenomena just under the center of a current-carrying flux tube and compared the threshold value with values calculated from experimental data. After such a comparison, we noted that at times the calculated FAC intensity exceeded the theoretically-defined threshold value that corresponded to the excitation of the low-frequency turbulence in the upper ionosphere. Finally, we interpreted the observed correlation between the appearance of aurora, PiB pulsation activity, and strong FACs through the mechanism related to the development of a quasi-oscillatory regime of anomalous resistivity and caused by the presence of the low-frequency turbulence on the auroral field lines.

\section{Observations}

The imaging riometer data was obtained from the Kilpisjärvi IRIS system in northern Finland (69.05 N, 20.79 E, L $\approx 6)$. The system operates at $38.2 \mathrm{MHz}$, and uses an array of 64 crossed half wave dipoles over a ground plane, with a set of Butler matrices from 49 independent beams. The signals are received by time-sharing into 7 riometers, the outputs of which are digitised (12 bits) every second. The time resolution is 1 second. The zenithal beam is $13^{\circ}$ wide between half power points and the best spatial resolution at $90 \mathrm{~km}$ is $20 \mathrm{~km}$. The oblique beams are considerably wider. The eighth riometer is connected to a wide-beam antenna at the site which covers a circle with a radius of $50 \mathrm{~km}$ at the $90 \mathrm{~km}$ level (Hargreaves et al., 1991, 1997; Detrick and Rosenberg, 1990).

The IMAGE project includes 15 ground-based magnetometers covering the range from sub-auroral through auroral lati-

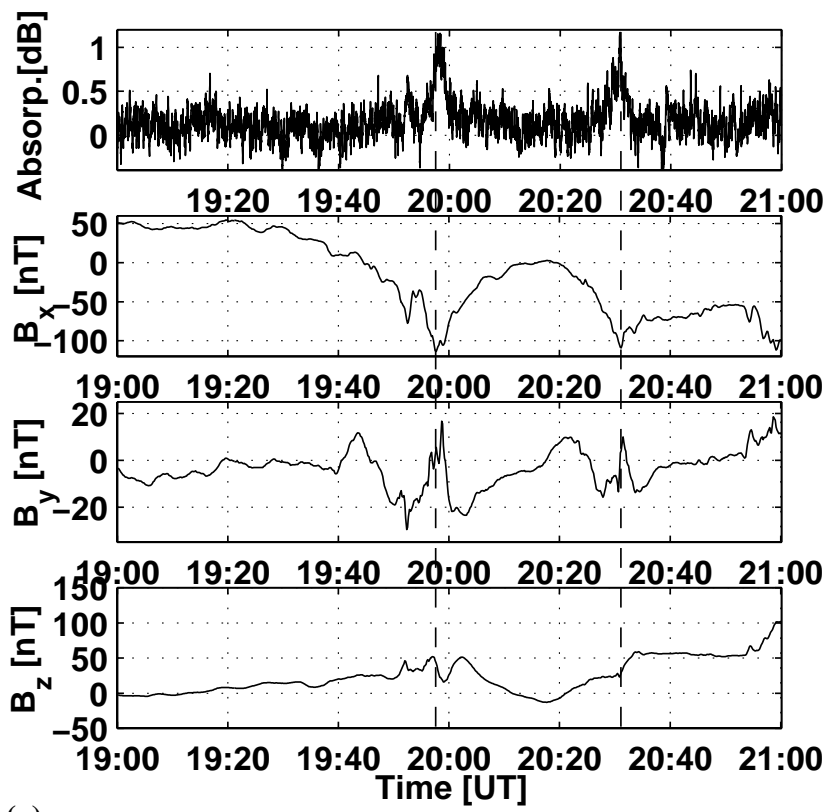

(a)

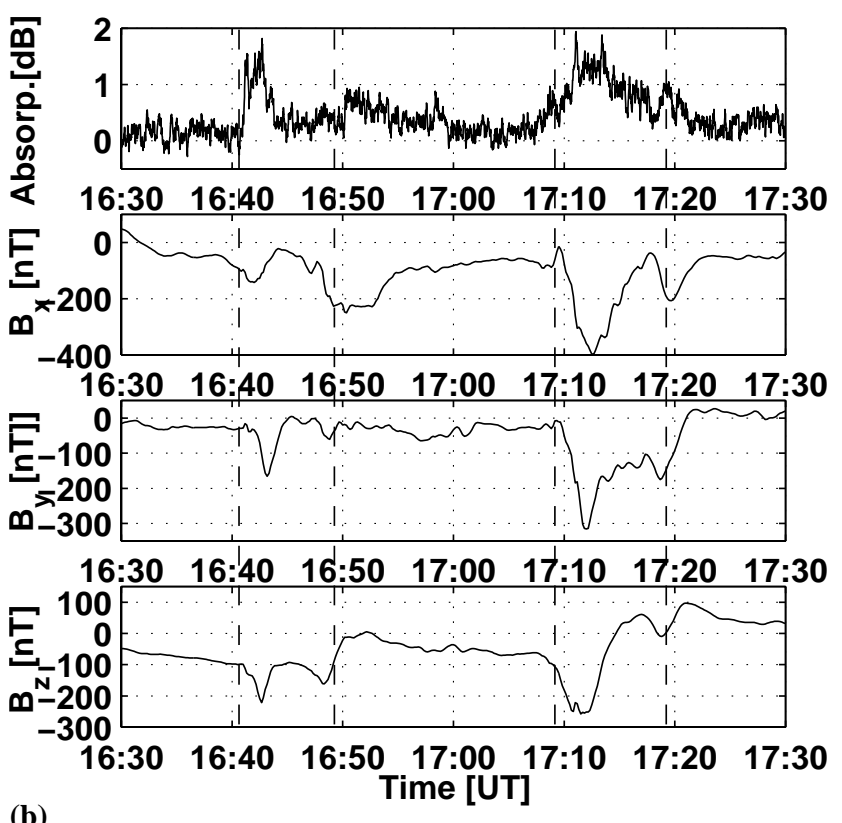

(b)

Fig. 1. The four panels in (a) and (b) above show (from the top) the wide-beam absorption data (IRIS) and the ground magnetic components (IMAGE) at Kilpisjärvi, Finland on 5 October 1999 from 19:00 to 21:00 UT and on 25 October 1999 from 16:30 to 17:30 UT. There are two different activations observed on 5 October 1999 at 19:57 and 20:30 UT, both in absorption and ground magnetic data. Note the clear signature of Pi2 pulsations in the magnetic Ycomponent from 19:55 to 20:00 UT! On 25 October 1999 there are four different activations observed at 16:41, 16:49, 17:09 and 17:19 UT.

tudes, up to the cusp/cleft regions in the European sector. All magnetometers meet certain standards in calibration, stability, and frequency response, ensuring that a homogeneous set of data emerges from the measurements. Samples are taken 
at 10 seconds intervals and the resolution of the instruments is $1 \mathrm{nT}$ or better (Lühr, 1994).

In this study we analyse the magnetic disturbances that were recorded by the IMAGE magnetometer network on 5 October 1999 from 19:00 to 21:00 UT, and on 25 October 1999 from 16:30 to 17:30 UT. We focused on local magnetometer measurements obtained at Kilpisjärvi. Multi-instrumental data, including riometer and ASC images, as well as pulsation magnetometer data from Kilpisjärvi were also added in order to study the spatial and temporal development of the phenomena involved. The selected time periods were characterized by disturbed geomagnetic conditions ( $A p=18$ on 5 October 1999 and $A p=16$ on 25 October 1999).

Figure 1 (panels from the top) shows relevant ground-based wide-beam absorption data from the imaging riometer IRIS and magnetograms ( $x$-, $y$ - and $z$-components) from the IMAGE station at Kilpisjärvi, respectively. From magnetometer data it can be seen that there are several activations observed at Kilpisjärvi on 5 October 1999 from 19:00 to 21:00 UT, namely at 19:57:40 and 20:30:10 UT, as well as on 25 October 1999 in the time interval from 16.30 to $17.30 \mathrm{UT}$, namely at 16:41, 16:49, 17:09, and 17:19 UT. On 5 October 1999, the two activation regions were located close to substorm onsets. However, among the activations on 25 October 1999, only the one at 17.09 UT marks the onset of an expansive phase of the substorm, as follows from the analysis of data from the whole IMAGE array (not shown here). Each activation is associated with an increase in riometer absorption and with a magnetic perturbation consistent with the development of filamentary upward FACs (seen in total equivalent current patterns (not shown here); the latter is also inferred from the behaviour of the $z$-component).

This interpretation is confirmed by displaying the Differential Equivalent Currents (DECs) with a differential time of $\Delta=30$ seconds. Figure 2 a-e shows the DEC vectors on 5 October 1999 at 19:57:40 and 20:30:10 UT, as well as on 25 October 1999 at $17: 10: 50$ to $17: 11: 10$ UT with the time resolution of 10 seconds. It follows from Fig. 2 that an anticlockwise current circulation around a center is exhibited when the current pattern is added to the former existing pattern. Such an equivalent current loop will be generated if a vertical upward directed FAC is escaping from a laterally homogeneous ionosphere (see, for example, Bösinger et al., 1981).

The corresponding activity, as seen by auroral the all-sky camera (ASC), shows a distinct increase in auroral luminosity around 19:57 and 20:30 UT on 5 October 1999 and shortly after 16:41, 16:46, 16:50 and 17:06 UT on 25 October 1999 (Figs. 3a, b, upper panel), whereas Figs. 4a, b presents the induction coil magnetometer data of the magnetic H-component during the same time interval, displaying pulsations in the $\mathrm{Pi} 2$ and Pi1B frequency range. PiB's are defined as broadband impulses. The long-period ( $T=40-150$ seconds) and the short-period ( $T=1-40$ seconds) parts of the impulses are usually specified by the abbreviations Pi2 and Pi1B, respectively (Saito, 1969; Kangas et al., 1979; Bösinger and Wedeken, 1987).
Comparing all activations and corresponding ionospheric effects, we note that the last activation at 17:19 UT, while it is also associated with the FAC, is accompanied by neither magnetic pulsations in the $\mathrm{PiB}$ range nor auroral activity.

There exists strong arguments that in order for a fieldaligned potential drop to build up enough strength to accelerate electrons to several hundred $\mathrm{eV}$ which is necessary for the excitation of auroral emissions, the FAC must exceed a critical value sufficient to cause plasma instabilities (Kindel and Kennel, 1971). We believe that it is the FAC intensity which is responsible for the complete set of phenomena observed just under the center of the current-carrying filament structure. It implies that the FAC intensity must exceed some threshold value. Both numerical and theoretical estimates of this value will be presented in the next section.

\section{Theoretical estimates}

We estimate first the magnitude of FAC in the upper ionosphere which is needed to produce aurora. As noted by Swift (1978), current-driven anomalous resistivity associated with plasma turbulence is most important where the electron drift velocity $V_{D}=j_{\|} / n e$ is maximal. To determine at which height this takes place, the calculation of the drift velocity was performed (Mozer et al., 1980) using a measured plasma density profile. In this, the fact that the current density $j_{\|}$ scales as the magnetic field strength $B$ is considered when the current in a flux tube is conserved. They found that the drift velocity peaks in the region of $6000-7000 \mathrm{~km}$. The corresponding FAC at this distance $\left(R \geq R_{E}\right)$ was estimated to be

$j_{\|}^{(m)} \simeq 1.3 \cdot 10^{-6} \mathrm{~A} / \mathrm{m}^{2}$

(Mozer et al., 1980).

For a dipole geomagnetic field $B(R)=B_{E} \cdot\left(R_{E} / R\right)^{3}$ (where $B_{E}$ is the magnetic field on the ground, at $R=R_{E}$ ), and using the conservation of magnetic flux and the total current in a magnetic flux tube, the relationship between the FAC density $j_{\|}^{(m)}$ in the magnetosphere at a distance $R_{m}$ and $j_{\|}^{(i)}$ in the ionosphere at $R_{i}$ can be obtained as follows: $j_{\|}^{(m)}=j_{\|}^{(i)} \cdot\left(R_{i} / R_{m}\right)^{3}$. Assuming that $R_{i} \sim R_{E}$ and $R_{m} \sim$ $2 R_{E}$, we obtain that the ionospheric FAC density is $j_{\|}^{(i)} \geq$ $5 \mu A / m^{2}$.

\section{Numerical estimates}

To obtain a representative figure, we used the formula given in the Appendix

$j_{\|}^{i}=\left(\frac{2 \Psi_{0}}{\mu_{0} R}\right)\left(\frac{a\left(2 a^{2}-r^{2}\right)}{\left(a^{2}+r^{2}\right)^{5 / 2}}\right)$

for the FACs associated with observed magnetic disturbances and absorption spikes. The magnetic permeability is $\mu_{0}=$ 

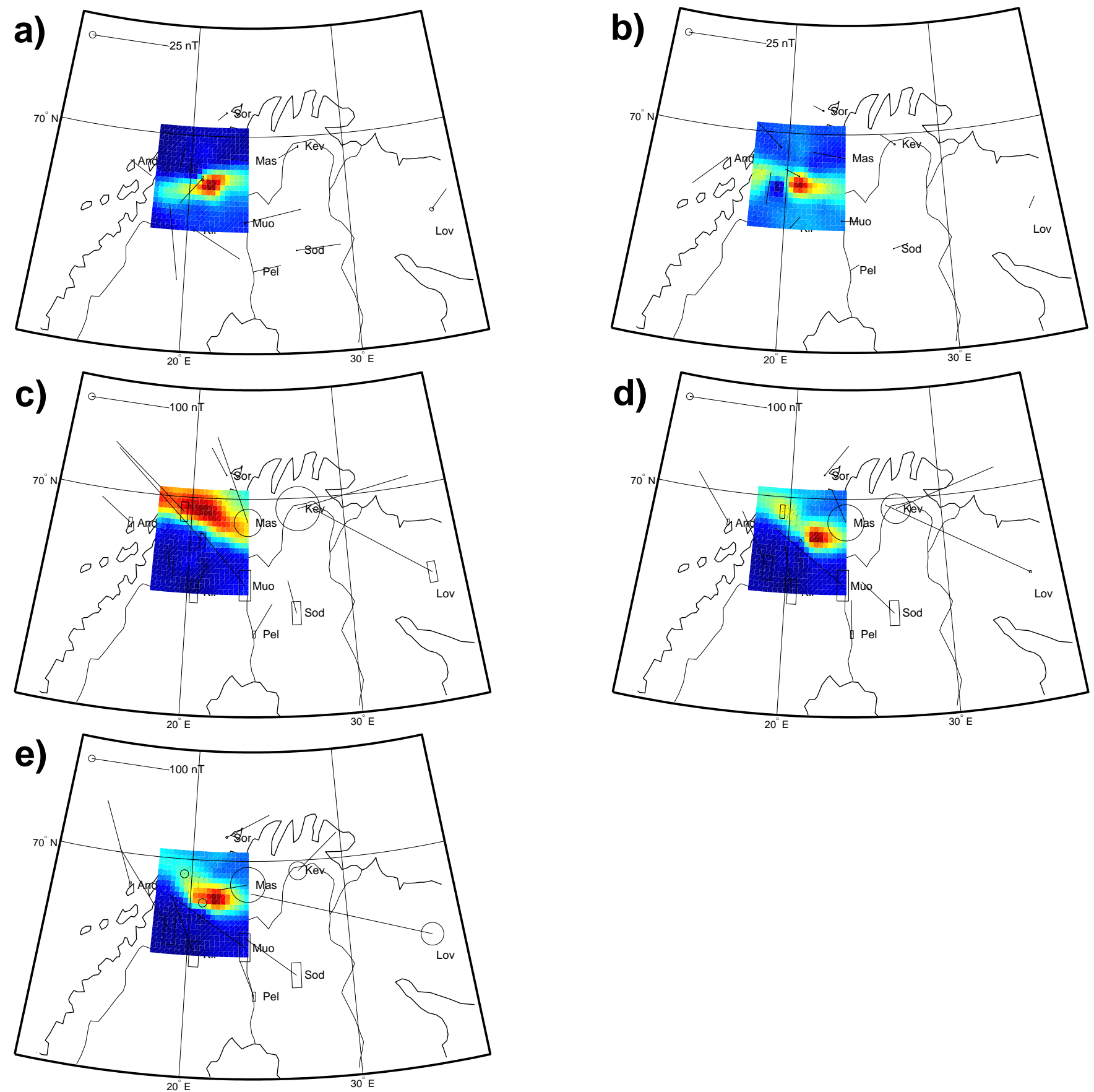

Fig. 2. Two-dimensional map of observations from the IMAGE network and the imaging riometer IRIS at Kilpisjärvi, Finland on 5 October 1999 from (a) 19:57:40 UT (b) 20:30:10 UT and (c-e) on 25 October 1999 from 17:10:50 to 17:11:10 UT with 10 second resolution. As a reference, a geographic grid, coastlines, and national borderlines are underlayed. The differential equivalent current $(\mathrm{DEC})$ vectors with $\Delta \mathrm{t}=$ 30 seconds show a counterclockwise and vortex-like pattern close by the region of an absorption spot near Kilpisjärvi. The vertical magnetic components, $b_{z}$, are represented by rectacular boxes and circles, whereas boxes are negative and circles are positive.

$4 \pi \cdot 10^{-7} \mathrm{H} / \mathrm{m}$ and $R=\Sigma_{H} / \Sigma_{P}$ is the Hall to Pedersen conductance ratio.

For this purpose we have to know the ratio $R$, the flux tube radius $a$, and the distance $r$ of a station from the center of a current-carrying filament. Walker and Bhatnagar (1989) have shown that the Hall and Pedersen conductances can be estimated from riometer data with the following empirical relations:

$$
\begin{aligned}
& \Sigma_{H}=1.49+16.7 \cdot A+0.15 \cdot A^{2}, \\
& \Sigma_{P}=0.60+4.57 \cdot A+0.07 \cdot A^{2},
\end{aligned}
$$



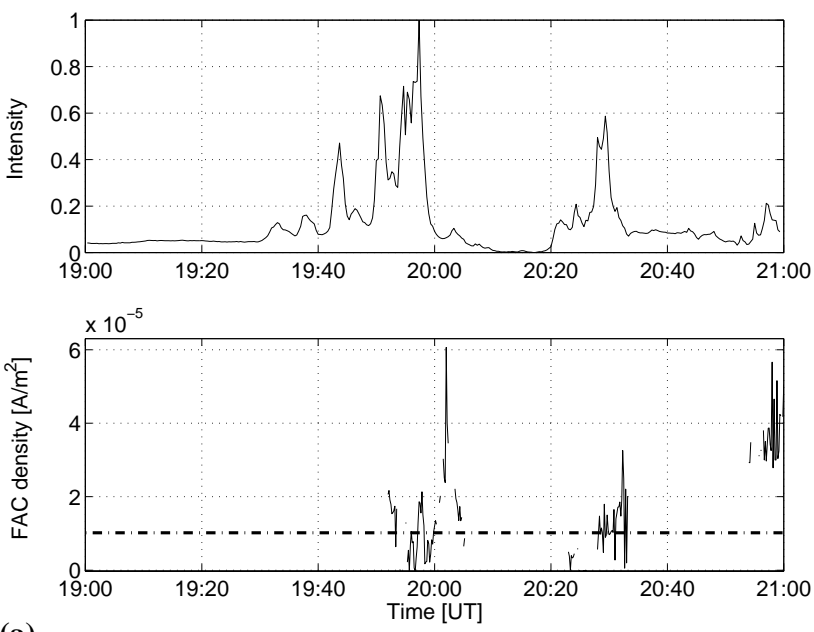

(a)
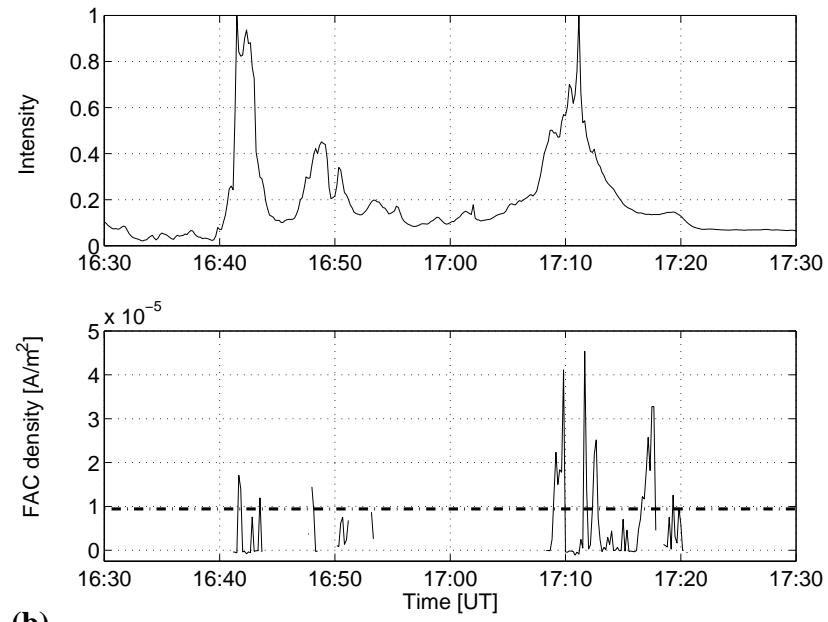

(b)

Fig. 3. The upper panel shows the normalized intensity from the ASC at Kilpisjärvi, Finland (a) on 5 October 1999 from 19:00 to 21:00 UT and (b) on 25 October 1999 from 16:30 to 17:30 UT with 20 seconds resolution. Regions of intense luminosity can be found for the same times as in Fig. 1. In the lower panel the fieldaligned current (FAC) density estimated by formula (8) from the appendix is shown. A dash-dotted line shows a theoretical estimated threshold value of $10^{-5} \mathrm{~A} / \mathrm{m}^{2}$. In (a) the strong activations exceed this value at 19:57 and 20:30 UT. The first three activations in (b) clearly reach this value. However, the last activation around 17:19 UT stays below this threshold value.

where $A$ is the ionospheric absorption at $30 \mathrm{MHz}$ in $\mathrm{dB}$. The absorption at frequency $f$ is given by Walker and Bhatnagar (1989):

$A(f) \approx A\left(\frac{30 \pm f_{L}}{f \pm f_{L}}\right)^{2}$,

where $f_{L}$ is the electron gyrofrequency (about $1.6 \mathrm{MHz}$ at 90 $\mathrm{km}$ altitude), and the plus/minus corresponds to ordinary/extraordinary mode wave. From these formulas we can estimate the value $R$ for absorption observed with the IRIS system at a frequency of $f=38.2 \mathrm{MHz}$ in our experimental data.
The radius $a$ and the distance $r$ can be calculated by an algorithm where the main points are as follows:

1. We select as a background level for the absorption the average value during the time interval under consideration (around $0.5 \mathrm{~dB}$ in our case);

2. For each absorption event above this level, the coordinates of maximum absorption, which are taken as the center of the current-carrying flux tube, can be determined (hence the distance $r$ from the observational point to the center of the tube can be determined);

3. The radius of the flux tube in the presented case is simply given as the half-width of the absorption patch image in the IRIS field of view. (For a more general approach, the morphology of the absorption filament can be taken into account.)

The geometry, size, and location of the absorption filament is used to estimate $j_{\|}$by formula 8 in the Appendix.

Taking all of these points into account, the current density during the selected time interval has been calculated, and the results are presented in Figs. 3a and $3 b$ (lower panel). The dashed line marks the threshold current density estimated in the previous section.

\section{Discussion and concluding comments}

The method used in the present study seems to be applicable for the estimation of FAC intensity associated with localized auroral structures detected within the field of view of both IRIS and ASC, provided there is no delay between auroral and absorption events (or at least the delay is not more than a few tens of seconds). In this case, the precipitations associated with aurora and absorption can approximately occupy the same ionospheric region and the current filament co-locates with the absorption spot which is generally not the case. For example, in a study using a network of broadbeam riometers and all-sky camera data, Collis and Korth (1983) reported a delay of about 2 min between the passage of a visible westward travelling surge and a sharp onset of radio absorption. Stocker at al. (1997) similarly observed the delay which varied from 0 to $60 \mathrm{sec}$.

As shown in Figs. 3a, b, the threshold value of FAC density measured at a given site is comparable to the estimated threshold level by each activation. In the case of the last activation on 25 October 1999 around 17:19 UT, only low auroral excitation is seen.

The estimated FAC density is, in general, equal or lower than the threshold value during this event. We suggest that part of the current can be attributed with high-energy particles which are responsible for the production of the absorption spike, while the other part of the current with a value which is probably less than the threshold can be attributed with relatively cold plasma, which is responsible for the welldeveloped region of anomalous resistivity and, as a consequence, the development of the aurora and $\mathrm{PiB}$ pulsations. 


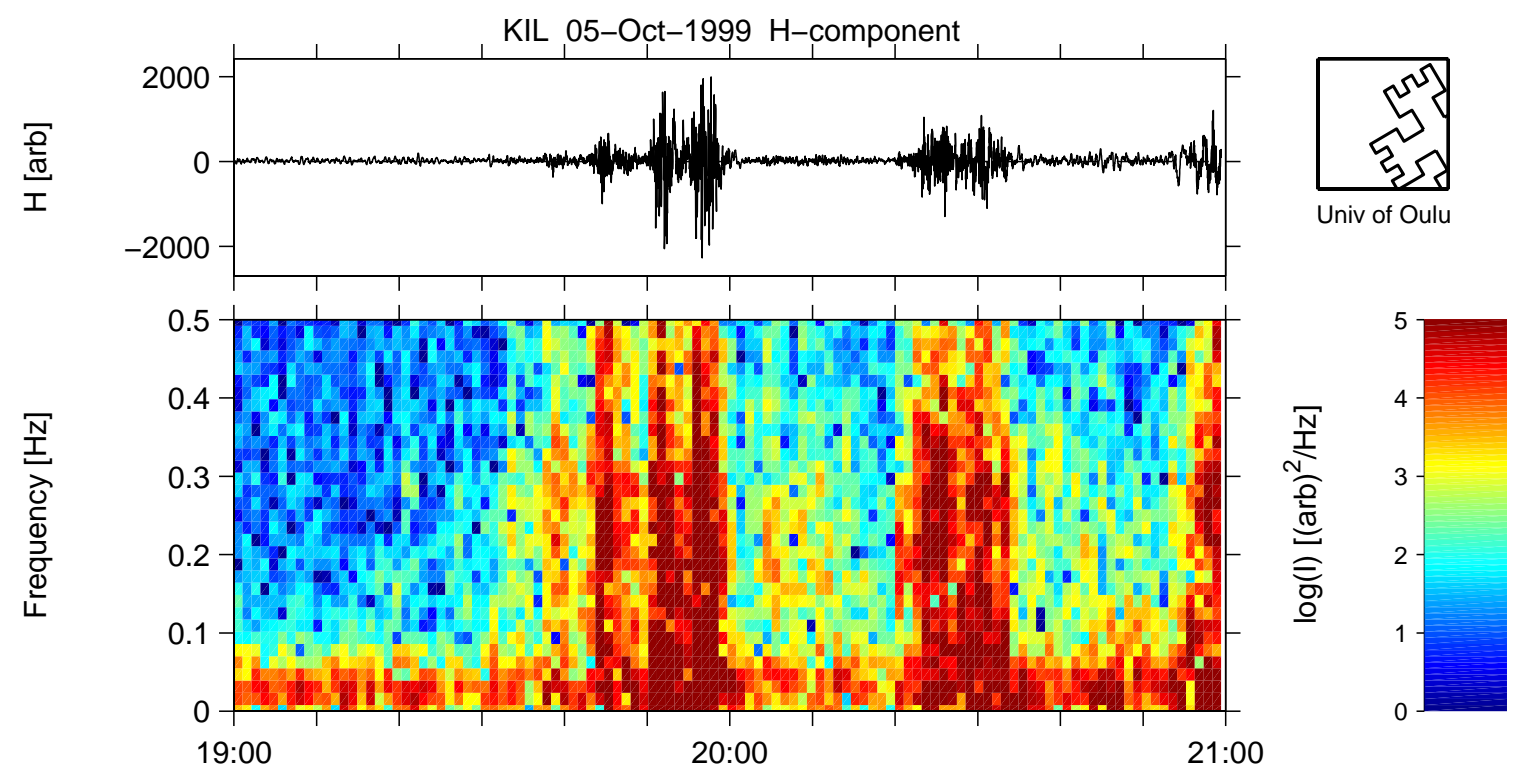

15-Jan-2001 11:25 NT user, FFT: 64/0, Sequence: 64/64

(a)

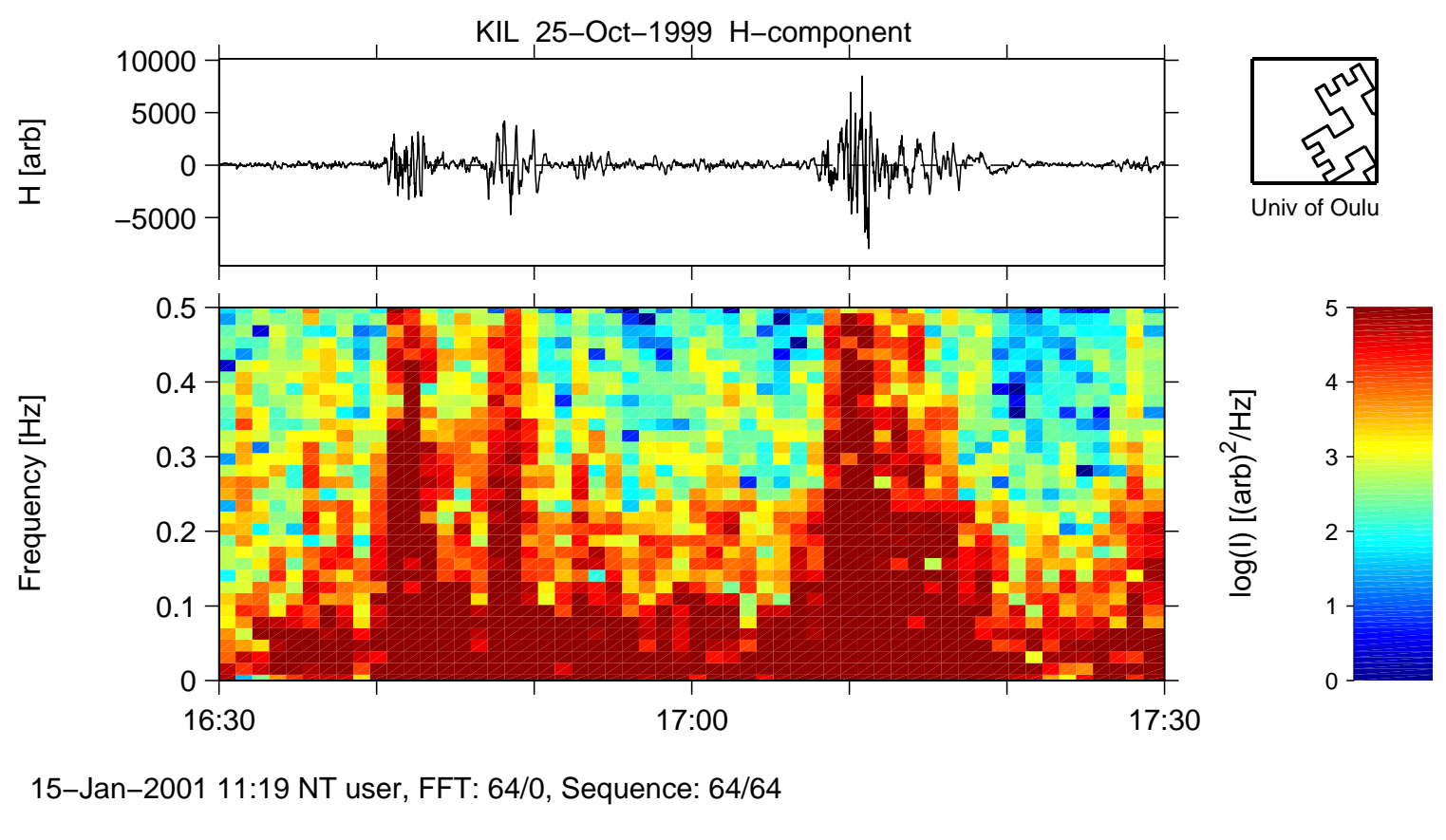

(b)

Fig. 4. The H-component of the pulsation magnetometer observations from Kilpisjärvi (a) on 5 October 1999 from 19:40 to 20:40 UT and (b) on 25 October 1999 from 16:30 to 17:30 UT is shown in the upper panel. Below, a frequency spectrum for the same time is shown. The four activation regions from the preview figures show significant Pi2 and Pib1 pulsations.

However, it is difficult to separate the relative contribution of the different plasma content into FAC.

Note that the absorption area shown by the IRIS data coincides with the local particle precipitation region, whereas the pulsation magnetometer responds to changes over a wide current region. Therefore, it may well be that the magnetic disturbance recorded at 17:19 UT originates in a strong current filament with its center far from Kilpisjärvi. If we check the complete IMAGE data set, it appears that the maximum magnetic disturbances were observed to the west from Kilpisjärvi at stations Tromso and Andenes.

Further, it is known that the Pi1B amplitude maximum observed on the ground is located just underneath active auroral forms (see, for example, Haldoupis et al., 1982; Bösinger and 
Wedeken, 1987; Arnold et al., 1998, and references therein). We computed the same relationship in our study (see Figs. $3 \mathrm{a}, \mathrm{b}$ and $4 \mathrm{a}, \mathrm{b})$. Note, however, the fact that the Pi1B activity maximum may not co-locate with the point of observation, which imposes an apparent limitation on our method.

Now, using the observed correlation of auroral and Pi1 pulsation activity in conjunction with strong FACs, we can produce theoretical arguments why aurora may be connected with the simultaneous appearance of Pi1 bursts. One of the possible explanations may be the following: we note that the value obtained for the magnetospheric current density $j_{\|}^{(m)}$ exceeded the threshold for the excitation of low-frequency turbulence in the topside ionosphere (see, for example, Mozer et al., 1980). The evolution of the corresponding ion-acoustic instability leads to quasi-oscillations around the saturation level with time approximately reciprocal to the instability growth rate $\tau_{1}$ and subsequent fading (in time $\tau_{2}$ which is 3-30 seconds for typical plasma parameters (Shalimov and Liperovsky, 1988; Pilipenko et al., 1999)). Consequent variations of anomalous conductivity result in pulsed electron precipitation and aurora, as well as noise generation in the Pi1B band.

It should be noted that, in principle, Pil bursts may be caused by the excitation of an Ionospheric Alfvén Resonator (IAR) with an impulse of FAC (Lysak, 1997). Preliminary theoretical estimates (Pilipenko et al., 1999) and experimental studies (Belyaev et al., 1999), however, show that the IAR resonance structure is very sensitive to energetic electron precipitations which may mask and/or prevent the resonance response of the ionosphere. In our data we may expect the presence of strong precipitations in the course of substorm development in association with the observed absorption spikes. However, just during these spikes, the most intense Pil bursts are developed in close association with FAC and aurora. Therefore, at least a part of the Pi1 activity can be associated with strong FAC development accompanied by quasi-oscillating anomalous resistivity.

\section{Appendix}

For the estimation of FAC intensity, we followed the idea suggested by Glassmeier and Heppner (1992) (see also Pilipenko et al., 1999). As a model of the current filament, they exploited the azimuthally symmetric flux tube, having a radius $a$, with an upward flowing FAC in the center and a downward flowing current at the flanks. The magnetic field in the atmosphere can be described by a magnetic scalar potential $\Psi(r, z)$ which obeys Laplace's equation. This potential in the atmosphere $(z>0)$ may be chosen to coincide with the potential of a point charge, $-4 \pi \Psi_{0} a$, located at $z=-a$. Thus, in cylindrical coordinates, the magnetic potential is $\Psi(r, z)=\Psi_{0}\left(a / \sqrt{(z+a)^{2}+r^{2}}\right)$ at $z>0$, where $\Psi_{0}$ is the amplitude factor. Then the ground magnetic field, $\mathbf{b}=-\left.\nabla \Psi\right|_{z=h}$, and the ionospheric FAC,

$j_{\|}=-\left.\left(\frac{2}{\mu_{0} R}\right) \nabla_{\perp}^{2} \Psi\right|_{z=0}$, are easily calculated (Glassmeier and Heppner (1992)). Here, $h$ is the height of the ionospheric layer, $\mu_{0}=4 \pi \cdot 10^{-7} \mathrm{H} / \mathrm{m}$ is the magnetic permeability, and $R=\Sigma_{H} / \Sigma_{P}$ is the Hall to Pedersen conductance ratio. The vertical magnetic component was chosen as a rough indicator of FAC intensity in the model. This component reaches its maximum value, $b_{z}^{\max }=\left(a /(a+h)^{2}\right) \Psi_{0}$, at the center of the current filament. This value for $b_{z}^{\max }$ is rarely measured. However, using ground vertical magnetic component $b_{z}$ measurements and following the suggestion by Pilipenko et al. (1999) we can obtain the amplitude factor $\Phi_{0}$

$\Phi_{0}=b_{z} \cdot \frac{\left((a+h)^{2}+r^{2}\right)^{3 / 2}}{a \cdot(a+h)}$,

for the case where the current-carrying flux tube is not direct above the magnetometer station. It was found that $\Phi_{0}$ is of the order of 5 to $10 \cdot 10^{-3} \mathrm{~T} \cdot \mathrm{m}$.

The FAC above the ionosphere

$j_{\|}=\left(\frac{2 \Psi_{0}}{\mu_{0} R}\right)\left(\frac{a\left(2 a^{2}-r^{2}\right)}{\left(a^{2}+r^{2}\right)^{5 / 2}}\right)$,

also reaches its maximum value, $j_{\|}^{\max }=\left(4 \Psi_{0} / \mu_{0} R\right)\left(1 / a^{2}\right)$, at $\mathrm{r}=0$.

Acknowledgement. Numerous discussions with T. Bösinger are greatly acknoledged. We acknowledge financial support from the Finnish Academy for Science and Letters. Without the IMAGE and ACS data provided by the Finnish Meteorological Institute and the IRIS riometer data provided by Lancaster University this study would have been not possible.

Topical Editor M. Lester thanks J. Wild and V. Pilipenko for their help in evaluating this paper.

\section{References}

Amm, O., Direct determination of the local ionospheric Hall conductance distribution from two-dimentional electric and magnetic field data: Application of the method using models of typical ionospheric electrodynamic situations, J. Geophys. Res., 100, 21473, 1995.

Arnold, R. L., Posch, J. L., Engebretson, M. J., Fukunishi, H., and Singer, H. J., Pil magnetic pulsations in space and at high latitudes on the ground, J. Geophys. Res., 103, 23581, 1998.

Belyaev, P. P., Bösinger, T., Isaev, S., and Kangas, J., First evidence at high latitudes for the ionospheric Alfvén resonator, J. Geophys. Res., 104, 4305, 1999.

Bösinger T., Alanko, K., Kangas, J., Opgenoorth, H., and Baumjohann, W. , Correlations between PiB type magnetic micropulsations, auroras and equivalent current structures during two isolated substorms, J. Atmos. Terr. Phys., 43, 933, 1981.

Bösinger, T. and Wedeken, U., Pi1B type magnetic pulsation simultaneously observed at mid and high latitudes, J. Atmos. Terr. Phys., 49, 573, 1987.

Collis, P. N. and Korth, A., Auroral radio absorption and westward travelling surge, Planet. Space Sci., 31, 1373, 1983.

Detrick, D. L. and Rosenberg, T. J., A phased-array radiowave imager for studies of cosmic noise absorption, Radio Sci., 25, 325, 1990. 
Fukushima, N., A generalized theorem of no groung magnetic effect of vertical current connected with Pedersen currents in the uniform conductivity ionosphere, Rep. Ionos. Space Res. Jap., 30, 35, 1976.

Glassmeier, K.-H. and Heppner, C., Traveling magnetospheric convection twin vortices: Another case study, global characteristics, and model, J. Geophys. Res., 97, 3977, 1992.

Haldoupis, C., Nielsen, E., Holtet, J. A., Egeland, A., and Chivers, H. A., Radar aurora observations during a bursts of irregular magnetic pulsations, J. Geophys. Res., 87, 1541, 1982.

Hargreaves, J. K., Detrick, D. L., and Rosenberg, T. J., Space-time structure of auroral absorption events observed with the imaging riometer at South Pole, Radio Sci., 26, 925, 1991.

Hargreaves, J. K., Browne, S., Ranta, H., Ranta, A., Rosenberg, T. J., and Detrick, D. L., A study of substorm-associated nightside spike events in auroral absorption using imaging riometers at South Pole and Kilpisjärvi, J. Atmos. Solar-Terr. Phys., 59, 853, 1997.

Inhester, B., Untiedt, J., Segatz, M., and Kurschner, M., Direct determination of the local ionospheric Hall conductance distribution from two-dimentional electric and magnetic field data, J. Geophys. Res., 97, 4073, 1992.

Kangas, J., Pikkarainen, T., Golikov, Y., Baransky, L., Troiskaya, V., and Sterlikova, V., Burst of Irregular Magnetic Pulsations, J. Geophys., 46, 237, 1979.

Kindel, J. M. and Kennel, C. F., Topside current instabilities, J. Geophys. Res., 76, 3055, 1971.

Lühr, H., The IMAGE magnetometer network, STEP International, USSCO, 4, 1994.

Lysak, R. L., Propagation of Alfvén waves through the ionosphere,
Phys. Chem. Earth, 22, 757, 1997.

Mende, S. B., Eather, R. H., Rees, M. H., Vondrak, R. R., and Robinson, R. M., Optical mapping of ionospheric conductances, J. Geophys. Res., 89, 1755, 1984.

Mozer, F. S., Cattell, C., Hudson, M. et al., Satellite measurements and theories of low altitude auroral particle acceleration, Space Sci. Rev., 27, 155, 1980.

Pilipenko, V. A., Shalimov, S. L., Fedorov, E. N., Engebretson, M. J., and Hughes, W. J., Coupling between field-aligned current impulses and Pi1 noise bursts, J. Geophys. Res., 104(8), 17419, 1999.

Robinson, R. M., Vondrak, R., Craven, J., Frank, L., and Miller, K., A comparison of ionospheric conductances and auroral luminosities observed simultaneously with the Chatanika Radar and the DE1 Auroral Imagers, J. Geophys. Res., 94, 5382, 1989.

Saito, T., Geomagnetic Pulsations, Space Sci. Rev., 15, 573, 1969.

Schlegel, K., Auroral zone E-region conductivities during solar minimum derived from EISCAT data, Ann. Geophysicae, 6, 129, 1988.

Stoker, P. H., Mathews, M. J., and Scourfield, M. W. J. , Cosmic radio noise absorption related to structures in auroral luminosity, J. Geophys. Res., 102, 7439, 1997.

Shalimov, S., and Liperovsky, V., On saturation of turbulent energy in field-aligned currents,Cosmic Res., 26, 247, 1988.

Swift, D. W., Mechanisms for the discrete aurora - a review, Space Sci. Rev., 22, 35, 1978.

Walker, J. K. and Bhatnagar, V. P., Ionospheric absorption, typical ionization, conductivity, and possible synoptic heating parameters in the upper atmosphere, J. Geophys. Res., 94, 3713, 1989. 